Chronic Obstructive Pulmonary Diseases: Journal of the COPD Foundation

\author{
Original Research
}

\title{
Symptom Burden and Medication Use Among Patients with Nontuberculous Mycobacterial Lung Disease
}

Vira Pravosud, MPH, MS ${ }^{1}$ David M. Mannino, MD ${ }^{1,2}$ Delia Prieto, MS ${ }^{2}$ Quan Zhang, PhD $^{3}$ Radmila Choate, $\mathrm{PhD}, \mathrm{MPH}^{1,2}$ Elisha Malanga, $\mathrm{BS}^{2}$ Timothy R. Aksamit, $\mathrm{MD}^{4}$

\begin{abstract}
Purpose: Respiratory diseases caused by nontuberculous mycobacteria (NTM) have become a significant concern for patients and health care providers. We aimed to compare symptoms experienced during the 2-week period, at a single point in time, by patients with NTM lung disease (NTMLD) who were currently on any medication to treat their NTMLD versus those not on any therapies.

Methods: We analyzed responses to a "Burden of NTM Survey" developed by the COPD Foundation. The study population included 266 individuals with NTMLD. Using adjusted penalized logistic regression models, we determined associations between the self-reported symptoms and the use of any medication to treat NTMLD.

Results: Based on available data, most respondents were aged 50 and older (95.1\%), of female gender (93.1\%), and had been living with NTMLD for more than 5 years (55.7\%). Many respondents reported symptoms that bother them very often or daily. After adjustment for age and gender, duration of living with NTMLD, and other respiratory illnesses, patients on medication had significantly larger odds of reporting difficulty in walking 500 meters without stopping, difficulty in interacting with others, fatigue or lack of energy, feelings of sadness or depression related to illness, and shortness of breath, wheezing or other difficulties.

Conclusion: In this study, patients currently on any medication to treat their NTMLD reported more symptoms associated with their NTMLD. Further investigations are needed to explore whether increased symptoms are related to differences in disease severity and/or medication effects.
\end{abstract}

\footnotetext{
Abbreviations: nontuberculous mycobacteria, NTM; NTM lung disease, NTMLD; mycobacterium avium complex, MAC; cystic fibrosis, CF; chronic obstructive pulmonary disease, COPD; analysis of variance, ANOVA; odds ratio, OR; adjusted odds ratio, $\mathbf{A O R}$; computed tomography, CT; confidence interval, CI

Funding Support: BronchandNTM360social was funded by a grant from Insmed Incorporated.

Date of Acceptance: February 17, 2021 | Published Online Date: February 19, 2021

Citation: Pravosud V, Mannino DM, Prieto D, et al. Symptom burden and medication use among patients with nontuberculous mycobacterial lung disease. Chronic Obstr Pulm Dis. 2021;8(2):243-254. doi: https://doi.org/10.15326/jcopdf.2020.0184
}

1 College of Public Health, University of Kentucky, Lexington, Kentucky, United States

2 COPD Foundation, Washington, DC, United States

3 School of Public Health, Rutgers University, Piscataway, New Jersey, United States

4 Pulmonary Disease and Critical Care Medicine, Mayo Clinic, Rochester, Minnesota, United States

\section{Keywords:}

nontuberculous mycobacteria; NTM; nontuberculous mycobacterial lung disease; NTMLD; BronchandNTM360social; Burden of NTM Survey

\section{Address correspondence to:}

Vira Pravosud, MPH, MS

College of Public Health

Department of Epidemiology

University of Kentucky

111 Washington Avenue, Room 211C

Lexington, KY 40536

Email: vira.pravosud@uky.edu

This article contains an online supplement. 


\section{Introduction}

The growing recognition of nontuberculous mycobacteria (NTM) as contributors to chronic lung infection and the increasing prevalence of NTM lung disease (NTMLD) has led to greater concern about their impact on patients, especially with other underlying pulmonary diseases. Among various clinical manifestations of NTM, pulmonary disease is the most frequent. ${ }^{1}$

Global increase in incidence and prevalence of NTMLD, as well as increased mortality rates due to NTMLD, have been widely discussed in the literature. $^{1-6}$ The following types of mycobacteria most commonly cause progressive NTMLD: the slowgrowing species, Mycobacterium avium complex (MAC) and Mycobacterium kasassii, as well as a rapid-growing group known as Mycobacterium abscessus. ${ }^{1,7}$ Any household or hospital water supply, mainly tap water or indoor showerhead, is considered a potential source of NTM. ${ }^{1,7}$ Of the 2 main species of MAC, Mycobacterium avium has been most closely associated with water exposures, whereas, Mycobacterium intracellulare is most associated with soil exposures.

Recent studies have demonstrated that immunocomprised individuals, including but not limited to people with HIV/AIDS, ${ }^{8}$ as well as thin postmenopausal women and the elderly ${ }^{9-11}$ can be at particular risk for NTMLD. The prevalence of NTM is also notable among patients with pre-existing respiratory illnesses, ${ }^{9,12,13}$ including persons with bronchiectasis, cystic fibrosis (CF), or chronic obstructive pulmonary disease (COPD). ${ }^{1,13-18}$

Previous studies conducted in the United States have found that place of residence also matters. People residing in the central, and especially in the southern and southeastern parts of the United States., may have a higher risk of exposure to NTM. ${ }^{1,2,7,19}$ More recently, particularly high rates of NTMLD have been identified in Hawaii. ${ }^{20}$ However, the existing data on prevalence and incidence rates of NTMLD in the United States are incomplete due to NTMLD not being reportable at a national level as well as at the state level in most states. ${ }^{5}$ The lack of active surveillance makes it difficult to determine the prevalence and incidence of NTMLD accurately. ${ }^{1,21}$ Ongoing studies are currently exploring more deeply the relationships between environmental exposures and the development of NTMLD.

\section{Nontuberculous Mycobacterial Lung Disease Symptomatology and Treatment}

NTM can lead to asymptomatic pulmonary infection and symptomatic illnesses. ${ }^{1}$ Evidence from previous studies has shown that NTMLD symptomatology varies. ${ }^{10,22,23}$ In general, symptoms are nonspecific and similar to symptoms attributed to often coexisting underlying pulmonary disorders. Signs and symptoms of the clinical presentation of NTMLD include but are not limited to cough, sputum production, dyspnea, hemoptysis, fever, night sweats, depression, fatigue, malaise, and weight loss. $1,10,22,23$

In patients with NTMLD, the decision of establishing treatment depends on the type of species, coexisting illnesses, and disease/symptom burden. ${ }^{1,13}$ Patients with MAC or Mycobacterium kansasii lung disease can benefit from multiple antibiotic and/or other mycobacterial therapies, whereas, those with Mycobacterium abscessus lung disease may also require surgery to remove localized areas of disease to increase the likelihood of complete cure. ${ }^{1}$ However, not every patient with an NTMLD-established diagnosis will require medication if, for instance, their disease progression is slow; and not every patient will benefit from NTM medication due to drug intolerance. ${ }^{1,13}$ In fact, because of the potential risks of serious adverse events, NTMLD treatment may not even be initiated or may need to be discontinued prematurely. ${ }^{1,13}$ An alternative treatment such as another medication or surgical procedure should be considered instead. ${ }^{1}$

Symptomatic improvements are one of the primary endpoints for monitoring of patients on NTM medication. ${ }^{1,13}$ Evidence shows that the duration to observe successful treatment outcomes with symptomatic improvements of NTMLD varies and may last from several months to several years. ${ }^{13}$ Although improvements in symptom severity while on medication are generally expected, ${ }^{1,13}$ some patients, especially those with other pulmonary comorbidities, may experience complications. ${ }^{1}$

\section{The Present Study}

Currently, there is little information about differences in NTMLD-related symptom burden among people who are or are not undergoing treatment for their NTMLD. The present study aimed to explore what symptoms are associated with NTMLD with or 
without the concurrent use of mycobacterial therapy.

For this investigation, we used responses to a "Burden of NTM Survey" developed by the COPD Foundation. The Foundation is a not-for-profit organization whose mission is to prevent and eliminate the progression of COPD. ${ }^{24}$ Created in 2004, the COPD foundation is developing and undertaking initiatives to educate and encourage the COPD community, and engage health care professionals, elective officials, and academic and industry leaders with the goal of COPD prevention and improvement of the quality of life of patients with COPD and related disorders. ${ }^{24}$ The 3 main areas of focus are (1) education and awareness, (2) public policy and advocacy, and (3) research. ${ }^{24}$

The "Burden of NTM Survey" was designed to better understand how much, and in what way, a patient may be affected by NTM lung infection or disease. A cohort of patients to survey was identified on the Bronchiectasis and NTM Initiative's 360social website. BronchandNTM360social is a free, interactive, online social community and network for patients with bronchiectasis and NTM, their family members, caregivers, and health care providers. ${ }^{25}$ It was also developed by and maintained by the COPD Foundation. The "Burden of NTM Survey" was posted on the BronchandNTM360social website. We aimed to determine the proportion of respondents of this survey with NTMLD, with or without NTM treatment, who self-reported symptoms. Results from this study will potentially assist with the future development of instruments for symptom surveillance of patients with NTMLD with and without the concurrent use of mycobacterial therapy.

\section{Methods}

\section{Study Design and Data Source}

The "Burden of NTM Survey" was made available online on the BronchandNTM360social website from September 12, 2016 through January 11, 2017. A total of 287 individuals completed the questionnaire. The collection of the informed consent was not required as the survey was optional and anonymous. This study qualified for exemption by the institutional review board at the University of Kentucky (\#17-0085-X6B).

We identified study participants with a self-reported NTMLD diagnosis through this cross-sectional survey. Participants were excluded if they had never been told by a doctor or a health care professional that they had an NTM lung infection or disease. The surveyed participants were asked to report whether they experienced 12 symptoms during the past 2 weeks (yes/no). Namely, those symptoms included: (1) cough, (2) a productive cough (i.e., coughing up blood, phlegm, or mucus), (3) fatigue or lack of energy, (4) sleep problems, (5) feelings of sadness or depression related to illness, (6) difficulty in walking 500 meters without stopping, (7) difficulty in interacting with others, (8) difficulty with sensitivity to cold or heat, (9) difficulty with fever, chills, or night sweats, (10) loss of appetite, (11) shortness of breath, wheezing or other breathing difficulties, and (12) body pain. The survey asked about the duration of living with an established diagnosis of NTMLD (less than 1 year, 1 to less than 2 years, 2 to 5 years, more than 5 years), whether participants considered themselves as having an ongoing NTM lung infection or disease (yes/no), current use of any medication(s) to treat their NTMLD (yes/no), duration of undergoing any medication(s) for NTMLD (less than 3 months, 4-6 months, 7-12 months, more than 12 months), and current experience of other lung illnesses such as asthma, bronchiectasis, COPD or emphysema, and CF. Participants could also list other pulmonary or nonpulmonary comorbid diseases in the "other, please specify" option. Demographic characteristics such as age category (less than 35 years, 35-49 years, 50-64 years, 65-79 years, and more than 80 years), gender, and place of residence (state and/or country as the survey was not limited only to patients residing in the United States) were obtained from the respondents.

\section{Statistical Analysis}

All statistical analyses were carried out using SAS, version 9.4 (SAS Institute Inc., Cary, North Carolina). Pearson $x^{2}$ (or Fisher's Exact Test, if the expected cell was $\leq 5$ ) was used to assess differences in categorical characteristics of the study sample by the 2 comparison groups: those with versus without NTM medications as well as to examine age- and gender-related differences among participants of the 4 U.S. regions (the Midwest, the Northeast, the South, and the West). We used Student's $t$-tests to assess significant differences in the mean number of selfreported symptoms within the 3 two-level, categorical variables describing age, gender, and the duration 
of NTMLD medication (for participants with any NTM therapies). The analyses of variance (ANOVA) followed by the Tukey's Studentized Range (HSD) post-hoc tests were used to determine differences in the mean number of self-reported symptoms between all pairs of the 3-level age variable as well as among the 4 residence groups for patients residing in the United States. Initially, we examined differences among the 3 age groups: (1) those who were less than 50 years old, (2) those who were 50 to less than 65 years old, and (3) those who were 65 years and older. However, evidence shows that NTMLD is more prevalent among people aged 50 years and older. ${ }^{11}$ Therefore, the survey participants were divided into 2 age categories for further comparison and adjustment in the regression modeling: (1) those who were younger than 50 years old, and (2) those aged 50 years and older.

Pearson $\mathrm{x}^{2}$ tests were conducted to identify differences in the percentages of individuals in the 2 groups (those who were versus those who were not currently on any medication to treat their NTMLD) having reported cough, fatigue or lack of energy, sleep problems, feelings of sadness or depression related to illness, difficulty in walking 500 meters without stopping, and difficulty in interacting with others. Single- and multivariable penalized logistic regression models with 95\% confidence intervals based on profile likelihood ${ }^{26}$ (as suggested, ${ }^{27}$ a superior method in small samples and sparse data ${ }^{28}$ ) were carried out to evaluate, respectively, unadjusted and adjusted, associations between self-reported symptoms (as separate outcomes of interest) and the use of any medication to treat NTMLD as the main exposure of interest. In multivariable modeling, the associations were adjusted for demographics (age and gender), duration of NTMLD, and having 1 or more of the respiratory illnesses such as asthma, bronchiectasis, CF, and COPD/emphysema. The survey participants could skip questions. We used complete case analysis with listwise deletion of missing data in the procedure steps for all types of comparison analyses. Two-sided $p$-values $<0.05$ were considered statistically significant.

\section{Results}

\section{Description of the Study Sample}

A total of 266 patients said that they had been told by a health care provider that they had an NTM lung infection or disease. Excluding missing data from the denominator for each variable of interest showed that most survey participants were aged 50 years or older (250, 95.1\%), of female gender (244, 93.1\%), and were living with NTMLD for more than 5 years (142, $55.7 \%$ ) (see Table 1). Half of the respondents reported being currently on medication for any NTMLD. As compared to participants without NTMLD therapies, a significantly larger proportion of those currently on any NTM medication reported living with NTMLD for less than a year or less than 2 years (respectively, $8.6 \%$ versus $1.6 \%$ and $17.2 \%$ versus $11.9 \%, p=0.0231$ ). The survey did not ask to specify the type of medication(s) nor mycobacteria or infection causing the disease; only 35 patients (13.2\%) could recall having been told by a health care provider that they had MAC/ Mycobacterium avium-intracellulare (MAI) using "other, please specify" option of the question about comorbid pulmonary conditions (results not shown in the table). Many of the participants who were versus who were not currently on any NTM medication were undergoing their therapies for more than a year (90,70.9\%); some were undergoing treatment for 7-12 months $(17,13.4 \%)$, and few were undergoing treatment for $4-6$ months $(8,6.3 \%)$ or less than 3 months (12, 9.5\%). Most of the survey participants who were without any NTM therapies skipped this question; only 1 person mentioned undergoing NTM treatment for more than 12 months. Many respondents $(247,92.9 \%)$ reported having other lung illnesses such as bronchiectasis (80.5\%), COPD or emphysema (29.7\%), asthma (24.6\%), or CF (2.6\%). A significantly larger proportion of participants who were versus who were not currently on any NTMLD medication reported having asthma (30.2\% versus $19.4 \%, p=0.0436)$ and COPD or emphysema (36.4\% versus $24.8 \%, p=0.0428$ ); however, more participants without any NTMLD medication reported having bronchiectasis $(76.7 \%$ versus $89.2 \%, p=0.0081$ ).

Two-thirds of respondents experienced half or more of the 12 listed symptoms (167, 66.3\%). As seen in Table 2 , the 3 most troubling symptoms were cough (61.0\%), fatigue or lack of energy (60.6\%), and shortness of breath, wheezing, or other breathing 


\section{Table 1. Characteristics of the Respondents of the "Burden of NTM Survey"

$\begin{array}{cccc}\text { Variables } & \text { All } & \text { With } & \text { Without } p \text {-value } \\ & (\mathrm{N}=266) & \text { Medication Medication } \\ 129,(50.0 \%) & 129,(50.0 \%)\end{array}$

\begin{tabular}{|c|c|c|c|c|}
\hline \multicolumn{4}{|l|}{ Age group $(n=263)$} & \multirow[t]{6}{*}{0.0584} \\
\hline$<35$ & $5(1.9)$ & $1(0.8)$ & $3(2.3)$ & \\
\hline $35-49$ & $8(3.0)$ & $7(5.5)$ & $0(0.0)$ & \\
\hline $50-64$ & 97 (36.9) & $48(37.5)$ & $47(36.7)$ & \\
\hline $65-79$ & $129(49.1)$ & $60(46.9)$ & $67(52.3)$ & \\
\hline$>80$ & $24(9.1)$ & $12(9.4)$ & $11(8.6)$ & \\
\hline \multicolumn{4}{|l|}{ Gender $(n=262)$} & \multirow[t]{3}{*}{0.1938} \\
\hline Female & $244(93.1)$ & $118(92.2)$ & $121(96.0)$ & \\
\hline Male & $18(6.9)$ & $10(7.8)$ & $5(4.0)$ & \\
\hline \multicolumn{4}{|l|}{ Duration of NTMLD ( $\mathrm{n}=255)$} & \multirow[t]{5}{*}{0.0231} \\
\hline$<1$ year & $13(5.1)$ & $11(8.6)$ & 2 (1.6) & \\
\hline 1 to less than 2 years & $37(14.5)$ & $22(17.2)$ & 15 (11.9) & \\
\hline 2 to 5 years & $63(24.7)$ & $26(20.3)$ & $37(29.4)$ & \\
\hline$>5$ years & $142(55.7)$ & $69(53.9)$ & $72(57.1)$ & \\
\hline \multicolumn{4}{|l|}{$\begin{array}{l}\text { Considering yourself as having an ongoing (or active, positive } \\
\text { sputum or lung culture) NTML infection or disease? }(\mathrm{n}=257)\end{array}$} & \multirow[t]{3}{*}{$<.0001$} \\
\hline No & $67(26.1)$ & $16(12.4)$ & $51(39.8)$ & \\
\hline Yes & $190(73.9)$ & $113(87.6)$ & $77(60.2)$ & \\
\hline \multicolumn{4}{|l|}{ Current use of any medications to treat the NTMLD $(n=258)$} & \multirow{3}{*}{--} \\
\hline No & $129(50.0)$ & -- & -- & \\
\hline Yes & $129(50.0)$ & -- & -- & \\
\hline \multicolumn{4}{|l|}{ Duration of undergoing NTMLD medications $(\mathrm{n}=128)$} & \multirow{5}{*}{--} \\
\hline$<3$ months & $12(9.4)$ & $12(9.5)$ & -- & \\
\hline 4-6 months & $8(6.3)$ & $8(6.3)$ & -- & \\
\hline 7-12 months & $17(13.3)$ & $17(13.4)$ & -- & \\
\hline$>12$ months & $91(71.1)$ & $90(70.9)$ & $1(0.8)^{b}$ & \\
\hline Surgery to resect localized areas of the lung to treat NTMLD $(n=254)$ & $39(15.4)$ & $22(13.3)$ & $17(17.5)$ & 0.3556 \\
\hline \multicolumn{4}{|l|}{ How long ago was the surgery done? $(\mathrm{n}=38)$} & \multirow[t]{5}{*}{0.5395} \\
\hline$<3$ months ago & $2(5.3)$ & $2(9.1)$ & $0(0.0)$ & \\
\hline 4-12 months ago & $6(15.8)$ & $4(18.2)$ & $2(2.5)$ & \\
\hline $1-3$ years ago & $8(21.1)$ & $3(13.6)$ & $5(3.4)$ & \\
\hline$>3$ years ago & $22(57.9)$ & $13(59.1)$ & $9(9.3)$ & \\
\hline \multicolumn{5}{|l|}{ Comorbid Respiratory Illnesses $(n=266)^{c}$} \\
\hline Bronchiectasis & $214(80.5)$ & 99 (76.7) & $115(89.2)$ & 0.0081 \\
\hline COPD/Emphysema & $79(29.7)$ & $47(36.4)$ & $32(24.8)$ & 0.0428 \\
\hline Cystic Fibrosis & $7(2.6)$ & $2(1.6)$ & $5(3.9)$ & 0.4467 \\
\hline Asthma & $64(24.1)$ & 39 (30.2) & 25 (19.4) & 0.0436 \\
\hline None of the above & $19(7.1)$ & $7(5.4)$ & $4(3.1)$ & 0.3552 \\
\hline
\end{tabular}

Numbers are presented as counts (n) and column percentages (\%) of those who responded. Missing values were excluded from the denominator; the number of participants included in the analysis is shown in parenthesis for each variable. Percentages may not add up to $100 \%$ because of rounding. Respondents could skip questions. Inconsistency in row totals for "All" versus "With and Without Medication" columns is accounted for unequal number of missing values for the total sample versus sub-sample of those who responded to the question about undergoing any NTM therapy (266 vs. 258, respectively). For example, among the 5 participants who were under 35 years old, only 4 responded to the question about NTM medication.

aFor comparison analyses (Pearson $\mathrm{x}^{2}$ or Fisher's Exact tests), listwise deletion of missing observations was performed for parameters with missing values bone participant who was not currently on any NTM therapy reported the duration of their NTM therapy that had been likely terminated or discontinued before the completion of the survey.

'Some patients reported having 1 or multiple comorbid respiratory illnesses and thus, the reported percentages exceed $100 \%$.

$\mathrm{NTM}=$ nontuberculous mycobacteria; NTMLD=nontuberculous mycobacterial lung disease; $\mathrm{COPD}=$ chronic obstructive pulmonary disease 
difficulties (51.2\%). For those who were on any NTMLD medication, the average number of selfreported symptoms did not vary significantly by the duration of undergoing any NTMLD treatment $(F=0.96, p=0.4161-$ results not shown in the table). Table 1 in the online supplement shows the percentages of those experiencing each self-reported symptom during the past 2 weeks by age and gender group. There were no significant age- or gender-related differences between the means of the number of selfreported symptoms (those less than 50 years versus those 50 years and older: $\mu=8.3$ versus $\mu=6.5, t=1.86$, $p=0.0638$; women versus men: $\mu=6.5$ versus $\mu=7.6$, $t=-1.36, p=0.1754$, see Figure 1 and Figure 2 in the online supplement, respectively). Of note, when treating the age variable as a 3-level variable (those less than 50 years versus those from 50 to less than 65 years versus those 65 years and older), we did not observe significant differences regarding the mean number of self-reported symptoms either $(\mu=$ 8.3 versus $\mu=6.7$ versus $\mu=6.4, F=1.93, p=0.1469$ results not shown in the table). Table 2 in the online supplement shows the percentages of self-reported symptoms by the region of residence for the subsample of 235 patients residing in the United States. Of note, participants of the 4 U.S. regions did not differ with respect to age or gender $\left(x^{2}=1.22, p=0.7490\right.$; $\mathrm{x}^{2}=2.68, p=0.4444$, respectively - results not shown in the table). The results of ANOVA suggested significant differences in the average number of selfreported symptoms among the 4 residence groups $(\mathrm{F}=2.77, p=0.0423)$. As seen in Figure 3 in the online supplement, patients from the Midwest reported a higher mean number of symptoms $(\mu=7.1)$, followed by patients from the South region $(\mu=6.8)$, the West $(\mu=6.5)$, and finally, from the Northeast region $(\mu=5.5)$. However, the post-hoc analysis did not reveal any statistically significant differences in pairwise comparisons among these 4 regions.

\section{Bivariate Results}

We observed significantly larger percentages of those who reported shortness of breath, wheezing or other breathing difficulties ( $80.0 \%$ versus $57.6 \%$, $p=0.0002)$, fatigue or lack of energy ( $87.9 \%$ versus $74.4 \%, p=0.0091$ ), feelings of sadness or depression related to illness ( $65.6 \%$ versus $45.2 \%, p=0.0014$ ), difficulty in walking 500 meters without stopping ( $48.8 \%$ versus $32.3 \%, p=0.0096)$, and difficulty in interacting with others (47.9\% versus $31.5 \%$, $p=0.0092$ ) among those who were currently taking any medication to treat their NTMLD as compared to those who were not on medication (see Table 3 ). The findings from the unadjusted logistic regression models were consistent with these results and revealed a significant association between being on medication and reporting of the 5 symptoms listed above (see Table 4).

\section{Table 2. Self-reported Symptoms Among the Respondents of the "Burden of NTM Survey"}

\begin{tabular}{|c|c|c|c|c|}
\hline \multirow[t]{2}{*}{ Symptom During the Past 2 Weeks } & \multicolumn{2}{|c|}{ Reporting Any } & \multicolumn{2}{|c|}{ Troubled Often or Daily } \\
\hline & $\mathrm{n}$ & $\%$ & $\mathrm{n}$ & $\%$ \\
\hline Fatigue or Lack of Energy ( $n=249$ ) & 202 & 81.1 & 151 & 60.6 \\
\hline Cough $(n=251)$ & 203 & 80.9 & 153 & 61.0 \\
\hline Shortness of Breath, Wheezing or Other Breathing Difficulties ( $\mathrm{n}=250$ ) & 172 & 68.8 & 128 & 51.2 \\
\hline Difficulty with Sensitivity to Cold or Heat $(n=242)$ & 155 & 64.1 & 100 & 41.3 \\
\hline Sleep Problems $(n=246)$ & 151 & 61.4 & 113 & 45.9 \\
\hline Cough up Blood, Phlegm, Mucus ( $\mathrm{n}=252)$ & 150 & 59.5 & 114 & 45.2 \\
\hline Feelings of Sadness or Depression Related to the Illness $(n=246)$ & 136 & 55.3 & 77 & 31.3 \\
\hline Experiencing Pain in Body $(n=243)$ & 120 & 49.4 & 90 & 37.0 \\
\hline Difficulty in Walking 500 Meters Without Stopping $(\mathrm{n}=247)$ & 100 & 40.5 & 79 & 32.0 \\
\hline Difficulty in Interacting With Others $(\mathrm{n}=245)$ & 97 & 39.6 & 64 & 26.1 \\
\hline Difficulty with Fever, Chills, or Night Sweats $(n=245)$ & 92 & 37.6 & 55 & 22.4 \\
\hline Loss of Appetite $(\mathrm{n}=247)$ & 86 & 34.8 & 59 & 23.9 \\
\hline
\end{tabular}

Numbers shown are counts $(n)$ and row percentages (\%) of those who responded. Missing values were excluded from the denominator. 


\section{Multivariable Results}

After adjusting for demographics, duration of living with NTM disease, and other respiratory illnesses, patients currently on medication, as compared to those who were not on medication, had significantly greater odds of reporting shortness of breath, wheezing or other difficulties (adjusted $O R,(A O R)=2.8,95 \%$ CI: 1.6-5.1), fatigue or lack of energy $(A O R=2.0,95 \% \mathrm{CI}$ :
1.1-4.1), feelings of sadness or depression related to illness ( $A O R=2.1,95 \%$ CI: 1.2-3.6), difficulty in walking 500 meters without stopping ( $\mathrm{AOR}=1.9,95 \%$ CI: 1.1-3.2), and difficulty in interacting with others ( $A O R=2.0,95 \%$ CI: 1.2-3.6). The odds of reporting cough and problems with sleep were similar in the 2 groups (see Table 4).

\section{Table 3. Comparison of Symptoms of the Respondents of the "Burden of NTM Survey": Those With Versus Without Any Medication to Treat Their Nontuberculous Mycobacterial Lung Disease}

\begin{tabular}{|c|c|c|c|c|c|}
\hline \multirow[t]{2}{*}{ Symptoms During the Past 2 Weeks } & \multicolumn{2}{|c|}{$\begin{array}{c}\text { With } \\
\text { Medication } \\
129(50.0 \%)\end{array}$} & \multicolumn{2}{|c|}{$\begin{array}{c}\text { Without } \\
\text { Medication } \\
129,(50.0 \%)\end{array}$} & \multirow[t]{2}{*}{$\begin{array}{c}p \text {-value } \\
\text { for } X^{2}\end{array}$} \\
\hline & $\mathrm{n}$ & $\%$ & $\mathrm{n}$ & $\%$ & \\
\hline Cough $(n=251)$ & 102 & 81.6 & 101 & 80.2 & 0.8727 \\
\hline Shortness of Breath, Wheezing or Other Breathing Difficulties $(n=250)$ & 100 & 80.0 & 72 & 57.6 & 0.0002 \\
\hline Fatigue or Lack of Energy $(n=249)$ & 109 & 87.9 & 93 & 74.4 & 0.0091 \\
\hline Sleep Problems $(\mathrm{n}=246)$ & 81 & 66.4 & 70 & 56.5 & 0.1177 \\
\hline Feelings of Sadness or Depression Related to the Illness ( $\mathrm{n}=246$ ) & 80 & 65.6 & 56 & 45.2 & 0.0014 \\
\hline Difficulty in Walking 500 Meters Without Stopping $(\mathrm{n}=247)$ & 60 & 48.8 & 40 & 32.3 & 0.0096 \\
\hline Difficulty in Interacting With Others $(\mathrm{n}=245)$ & 58 & 47.9 & 39 & 31.5 & 0.0092 \\
\hline
\end{tabular}

\section{Table 4. Associations Between 7 Self-reported Symptoms (Outcomes) and the Use of Medication to Treat Nontuberculous Mycobacterial Lung Disease ${ }^{a}$}

\begin{tabular}{|c|c|c|c|c|c|c|c|c|}
\hline \multirow[t]{2}{*}{ Symptoms } & \multicolumn{4}{|c|}{ Unadjusted Results } & \multicolumn{4}{|c|}{ Adjusted Results } \\
\hline & $n^{b}$ & OR & $95 \% \mathrm{Cl}$ & $p$-value & $\mathrm{n}^{\mathrm{c}}$ & AOR & $95 \% \mathrm{Cl}$ & $p$-value \\
\hline Cough & 251 & 1.1 & $0.6-2.1$ & 0.7748 & 243 & 1.0 & $0.5-1.9$ & 0.9807 \\
\hline Shortness of Breath, Wheezing or Other Breathing Difficulties & 250 & 2.9 & $1.7-5.2$ & 0.0002 & 243 & 2.8 & $1.6-5.1$ & 0.0006 \\
\hline Fatigue or Lack of Energy & 249 & 2.5 & $1.3-4.9$ & 0.0084 & 242 & 2.0 & $1.1-4.1$ & 0.0370 \\
\hline Sleep Problems & 246 & 1.5 & $0.9-2.5$ & 0.1132 & 239 & 1.7 & $1.0-2.9$ & 0.0591 \\
\hline Feelings of Sadness or Depression Related to Illness & 246 & 2.3 & 1.4-3.9 & 0.0015 & 240 & 2.1 & $1.2-3.6$ & 0.0062 \\
\hline Difficulty in Walking 500 Meters Without Stopping & 247 & 2.0 & $1.2-3.3$ & 0.0091 & 239 & 1.9 & $1.1-3.2$ & 0.0256 \\
\hline Difficulty in Interacting with Others & 245 & 2.0 & $1.2-3.4$ & 0.0093 & 238 & 2.0 & $1.2-3.6$ & 0.0126 \\
\hline
\end{tabular}

aBased on the responses of the "Burden of NTM Survey"

Results from single and multivariable penalized logistic regressions. In the multivariable modeling, the results are adjusted for age, gender, duration of NTMLD, and having reported one or more of other respiratory illnesses such as asthma, bronchiectasis, CF, and COPD/emphysema.

${ }^{b, c}$ Numbers shown are the final number of observations included in each of the 7 single- and multivariable models $\left(\mathrm{n}^{\mathrm{b}}\right.$ and $\mathrm{n}^{\mathrm{c}}$ respectively).

$\mathrm{NTM}=$ nontuberculous mycobacteria; $\mathrm{NTMLD}=$ nontuberculous mycobacterial lung disease; $\mathrm{COPD}=$ chronic obstructive pulmonary disease; $\mathrm{CF}=$ cystic fibrosis; $\mathrm{OR}=$ odds ratio; $\mathrm{AOR}=$ adjusted odds ratio; $\mathrm{CI}=$ confidence interval 


\section{Discussion}

In the United States and throughout many areas of the world, increasing prevalence rates for NTM respiratory infections have become a concern for patients and health care providers. ${ }^{7,11,17,19}$ NTM may cause both asymptomatic lung infection and symptomatic disease. ${ }^{1}$ Resolution of or even improvements in symptoms is an important endpoint for therapeutic drug monitoring of patients with NTM therapies. ${ }^{13}$ This cross-sectional study describes a population of individuals with a self-reported NTMLD with or without NTM treatment and associated symptoms. Many respondents reported having at least 1 comorbid pulmonary illness in addition to their NTMLD confirming at-risk populations. Understanding the burden of NTMLD may ultimately assist caregivers in focusing on symptom management in addition to impact of therapeutic intervention.

As one of the priorities of the patient-centered research for NTM lung infection and disease, ${ }^{29}$ development of an NTMLD severity assessment tool has become a topic of emerging discussions. ${ }^{29,30}$ Due to the lack of active surveillance, NTMLD is underinvestigated, and the outcomes of NTM treatment are not well understood. Undergoing NTM therapies, which can last on average from 18-24 months, ${ }^{1}$ may not guarantee successful treatment results. ${ }^{1}$ Previous studies have shown evidence of NTM reinfection and recurrence among patients on NTM medication. ${ }^{31-33}$ Given a long-term duration of living with NTMLD (which some may posit as a chronic condition), ${ }^{9}$ development of a validated composite score of NTMLD-related symptom burden and a measure of disease severity is very important. This knowledge can improve the monitoring of existing and assessment of new NTM therapies. ${ }^{29,30,34}$ Similar to assessment tools for patients with $\mathrm{CF}^{35}$ and bronchiectasis, ${ }^{36,37}$ Quittner et al created a healthrelated quality of life module and a draft measure specifically for NTM. ${ }^{30}$ Based on the concerns reported by patients and pulmonologists, the module included NTM and digestive symptoms, body image and eating problems. ${ }^{30}$ Quittner et al also proposed an algorithm for administering the NTM module to accompany assessment tools for patients with CF and bronchiectasis. ${ }^{38}$ More data collection and research is needed for further development of the module's themes and their validation. ${ }^{30,38}$

\section{Principal Findings}

To our knowledge, this is the first study in the United States that examined associations of symptoms of patients with self-reported NTMLD with and without the use of NTM medication. More than half of the surveyed participants reported living with an NTMLD for more than 5 years, highlighting the chronic nature of NTMLDs. ${ }^{17,39-41}$ In contrast to earlier studies noting a predominantly male distribution in those with NTM infection, our study is in alignment with recent research characterizing NTMLD predominately in women and older (those aged 50 years or older) patients. ${ }^{42}$

The findings revealed that experiencing symptoms was common among NTMLD patients with and without treatment. The 3 most troubling symptoms were cough, fatigue or lack of energy, and shortness of breath, wheezing or other breathing difficulties. After adjusting for demographics, duration of living with an NTMLD, and other respiratory disorders, we observed a greater burden of symptoms among those receiving NTM medication. Participants with NTM therapies were more likely to report shortness of breath, wheezing or other breathing difficulties, fatigue or lack of energy, feelings of sadness or depression related to illness, difficulty in walking 500 meters without stopping, and difficulty in interacting with others. The present study, however, may be subject to confounding by indication. Since NTMLD severity is one of the factors influencing the initiation of NTM treatment, ${ }^{1}$ it is likely that NTM medications were prescribed to patients with a greater disease severity and symptom burden.

Our study could not determine why eligible participants who supposedly had NTMLD were without any NTM therapies at the time of the survey. It is possible that participants who were not on NTM medication had asymptomatic NTM infection confirmed by skin and/or antibody tests, ${ }^{1}$ or that they had been successfully treated in the past, and thus, no longer required NTM treatment. Contrasting answers to the questions about the duration of living with NTMLD and duration of NTM medication (which was missing for those currently not on medication) could have provided more explanations. Other reasons for not undergoing NTM treatment could be related to either drug non-adherence or drug intolerance. ${ }^{13}$ Many participants without NTM medication (60.2\%) reported considering themselves as having an 
ongoing NTMLD, which was likely an indication of non-negligible symptom burden among them. A significant reason, such as drug intolerance, could have influenced the decision of not establishing or halting NTM medication for such individuals. Indeed, compared to those with any NTM therapy, more (although not statistically significantly) respondents without NTM therapies reported undergoing surgery to treat their NTMLD, which is important given that surgery could be one of the alternative treatments for NTMLD patients with severe NTM drug intolerance. ${ }^{1}$ Future research should include follow-up questions for respondents without NTM therapies to determine reasons for not initiating or stopping NTM treatment and its impact on symptom burden.

\section{Limitations}

In the present study, the information about specific mycobacteria or infection causing the disease was not available, and data on what type of treatment was used to treat patients' NTMLD were also limited. Future similar studies should include NTM species identification to the questionnaires as this can explain the choice for NTM medication. ${ }^{1}$ To establish successful treatment outcomes, it is critically important to fully evaluate, correctly diagnose, and treat NTMLD and other co-existing lung diseases which are common and are often difficult to manage. ${ }^{28}$ Although NTM therapies are presumed to help with symptoms, they can have substantial side effects (including but not limited to gastrointestinal, hearing, and visual effects). ${ }^{13}$ Recently published results from a 10-year retrospective cohort study from Italy showed that approximately $40 \%$ of NTMLD patients had experienced adverse effects from NTM medication, among whom almost onethird of patients could not continue their therapies. ${ }^{43}$ The effect of unmeasured confounding in our study, due to unknowing what kind of treatment was used and for which NTM species, could be significant. Not accounting for other co-existing lung disease medication and its impact on patient's symptoms can raise similar issues.

Furthermore, this was a cross-sectional survey, and no longitudinal data were collected. The present study could not assess the effect of treatment on symptoms over time, nor did the survey ask the sequence number of the NTM diagnosis or treatment, which is a substantial limitation given the possibility of reinfection and recurrence of NTMLD. ${ }^{31-33}$ The study used a convenience sample of people associated with the BronchandNTM360social, and thus, there is a possibility of selection bias that might have led to over-representation of participants with some demographic characteristics or with co-existing pulmonary disorders. No causal inferences should be applied to the general population.

Finally, the identification of NTMLD is not always straightforward. Establishing a diagnosis of NTMLD for patients with pulmonary symptoms requires 3 criteria: (1) a compatible chest radiograph or chest high-resolution computed tomography (CT) scan, (2) a minimum of 2 positive sputum cultures for nontuberculous acid-fast bacilli or 1 positive bronchial wash/lavage or 1 positive biopsy culture, and finally, (3) the exclusion of other diseases to account for symptoms and radiographic abnormalities. ${ }^{1}$ Our analysis was based on self-reported data; the diagnosis of NTM was not confirmed. Our study did not use any standard measures of disease severity such as CT scan results or a pulmonary function test to account for differences in symptomatology observed in NTMLD patients with versus without NTMLD medication. Previous research has shown significantly greater declines in forced expiratory volume in 1 second and forced vital capacity among unsuccessfully treated patients with NTMLD as compared to those without NTMLD treatment or those with successful treatment outcomes. $^{44}$

Despite its limitations, the present study is a good example of a research initiative that can be undertaken in the absence of any ongoing surveillance. The investigators can no longer explain global growth in prevalence and incidence rates of NTMLD by advanced diagnostic procedures only. ${ }^{45}$ We aimed to better understand the burden of NTMLD-related symptoms. Our findings showed how common symptoms were and how often they had troubled patients. Further investigations are needed to explore whether increased symptoms are related to differences in disease severity and/or medication effects.

\section{Conclusion}

Patients currently on any medication to treat their NTMLD reported more symptoms associated with their NTMLD. Although more research is still needed, 
the present study provides insights that may help with the development of a practical instrument (e.g., a composite symptom score) for symptoms surveillance and assessment of the burden of NTMLD.

\section{Acknowledgements}

Author contributions: VP conducted data analysis and interpretation, drafted and revised the manuscript. DM, VP, DP, EM, QZ, TA, RC provided substantial contributions to the concept and design. RC, DM, QZ, TA, DP, EM critically revised the manuscript for important intellectual content. DM provided supervision, administrative, technical, and material support. DP and EM were involved in obtaining the funding.
The NTM survey was developed by the COPD Foundation with input from Amy Leitman and Susan Wisliceny from NTM Info \& Research and from Insmed Incorporated. The findings achieved herein are solely the responsibility of the authors.

\section{Declaration of Interest}

The authors report no conflicts of interest in this work. VP served as a Research Graduate Assistant at the University of Kentucky and assisted the COPD Foundation analysis team during this project. DM, DP and EM are staff members of the COPD Foundation. $\mathrm{QZ}$ is a former employee of Insmed Incorporated. RC is a Research Assistant Professor at the University of Kentucky. TA serves as the Chair of the COPD Foundation's Bronchiectasis and NTM Research Registry. 


\section{References}

1. Griffith DE, Aksamit T, Brown-Elliott BA, et al. An official ATS/IDSA statement: diagnosis, treatment, and prevention of nontuberculous mycobacterial diseases. Am J Respir Crit Care Med. 2007;175(4):367416. doi: https://doi.org/10.1164/rccm.200604-571ST

2. Honda JR, Bernhard JN, Chan ED. Natural disasters and nontuberculous mycobacteria: a recipe for increased disease? Chest. 2015;147(2):304-308. doi: https://doi.org/10.1378/chest.14-0974

3. Thomson R, NTM working group at Queensland TB Control Centre and Queensland Mycobacterial Reference Laboratory. Changing epidemiology of pulmonary nontuberculous mycobacteria infections. Emerg Infect Dis. 2010;16(10):1576-1583. doi: https://doi.org/10.3201/eid1610.091201

4. Kendall BA, Winthrop KL. Update on the epidemiology of pulmonary nontuberculous mycobacterial infections. Semin Respir Crit Care Med. 2013;34(1):87-94. doi: https://doi.org/10.1055/s-0033-1333567

5. Prevots DR, Marras TK. Epidemiology of human pulmonary infection with nontuberculous mycobacteria. Clin Chest Med. 2015;36(1):1334. doi: https://doi.org/10.1016/j.ccm.2014.10.002

6. Morimoto K, Iwai $\mathrm{K}$, Uchimura $\mathrm{K}$, et al. A steady increase in nontuberculous mycobacteriosis mortality and estimated prevalence in Japan. Ann Am Thorac Soc. 2014;11(1):1-8.

doi: https://doi.org/10.1513/AnnalsATS.201303-067OC

7. Johnson MM, Odell JA. Nontuberculous mycobacterial pulmonary infections. J Thorac Dis. 2014;6(3):210-220.

doi: $10.3978 /$ j.issn.2072-1439.2013.12.24

8. Falkinham JO. Epidemiology of infection by nontuberculous mycobacteria. Clin Microbiol Rev. 1996;9(2):177-215. doi: https://doi.org/10.1128/CMR.9.2.177

9. Chan $\mathrm{ED}$, Iseman $\mathrm{MD}$. Slender, older women appear to be more susceptible to nontuberculous mycobacterial lung disease. Gend Med. 2010;7(1):5-18. doi: https://doi.org/10.1016/j.genm.2010.01.005

10. Kim RD, Greenberg DE, Ehrmantraut ME, et al. Pulmonary nontuberculous mycobacterial disease. Am J Respir Crit Care Med. 2008;178(10):1066-1074.

doi: https://doi.org/10.1164/rccm.200805-686OC

11. Cassidy PM, Hedberg K, Saulson A, McNelly E, Winthrop KL. Nontuberculous mycobacterial disease prevalence and risk factors: a changing epidemiology. Clin Infect Dis. 2009;49(12):e124-e129. doi: https://doi.org/10.1086/648443

12. Ford ES, Horne DJ, Shah JA, Wallis CK, Fang FC, Hawn TR. Speciesspecific risk factors, treatment decisions, and clinical outcomes for laboratory isolates of less common nontuberculous mycobacteria in Washington state. Ann Am Thorac Soc. 2017;14(7):1129-1138. doi: https://doi.org/10.1513/AnnalsATS.201609-731OC
13. Haworth CS, Banks J, Capstick T, et al. British Thoracic Society guidelines for the management of non-tuberculous mycobacterial pulmonary disease (NTM-PD). Thorax. 2017;72(Suppl 2):ii1-ii64. doi: https://doi.org/10.1136/thoraxjnl-2017-210927

14. Olivier KN, Weber DJ, Richard J. Wallace J, et al. Nontuberculous mycobacteria. Am J Respir Crit Care Med. 2003;167(6):828-834. doi: https://doi.org/10.1164/rccm.200207-6780C

15. Roux A-L, Catherinot E, Ripoll F, et al. Multicenter study of prevalence of nontuberculous mycobacteria in patients with cystic fibrosis in France. J Clin Microbiol. 2009;47(12):4124-4128. doi: https://doi.org/10.1128/JCM.01257-09

16. Qvist T, Gilljam M, Jönsson B, et al. Epidemiology of nontuberculous mycobacteria among patients with cystic fibrosis in Scandinavia. $J$ Cyst Fibros. 2015;14(1):46-52. doi: https://doi.org/10.1016/j.jcf.2014.08.002

17. Prevots DR, Shaw PA, Strickland D, et al. Nontuberculous mycobacterial lung disease prevalence at four integrated health care delivery systems. Am J Respir Crit Care Med. 2010;182(7):970-976. doi: https://doi.org/10.1164/rccm.201002-03100C

18. Aksamit TR. Mycobacterium avium complex pulmonary disease in patients with pre-existinglung disease. Clin ChestMed. 2002;23(3):643653. doi: https://doi.org/10.1016/S0272-5231(02)00022-9

19. Adjemian J, Olivier KN, Seitz AE, et al. Spatial clusters of nontuberculous mycobacterial lung disease in the United States. Am J Respir Crit Care Med. 2012;186(6):553-558. doi: https://doi.org/10.1164/rccm.201205-0913OC

20. Adjemian J, Olivier KN, Seitz AE, Holland SM, Prevots DR. Prevalence of nontuberculous mycobacterial lung disease in U.S. Medicare beneficiaries. Am J Respir Crit Care Med. 2012;185(8):881-886. doi: https://doi.org/10.1164/rccm.201111-2016OC

21. Aksamit TR, Philley JV, Griffith DE. Nontuberculous mycobacterial (NTM) lung disease: the top ten essentials. Respir Med. 2014;108(3):417-425.

doi: https://doi.org/10.1016/j.rmed.2013.09.014

22. Prince DSMD, Peterson DDMD, Steiner RMMD, et al. Infection with mycobacterium avium complex in patients without predisposing conditions. N Engl J Med. 1989;321(13):863-868. doi: https://doi.org/10.1056/NEJM198909283211304

23. Reich JM, Johnson RE. Mycobacterium avium complex pulmonary disease presenting as an isolated lingular or middle lobe pattern: the Lady Windermere Syndrome. Chest. 1992;101(6):1605-1609. doi: https://doi.org/10.1378/chest.101.6.1605

24. Walsh JW. The evolving role of COPD patient advocacy organizations for COPD. J Thorac Dis. 2012;4(6):676-680. doi: 10.3978/j.issn.2072-1439.2012.10.03

25. COPD Foundation. BronchandNTM360social. BronchandNTM360social website. Published 2019. Accessed Jul 1, 2020. https://www. bronchiectasisandntminitiative.org/BronchandNTM360social/ Community-Discussions /Activity-Feed/?lastItem=5972 
26. Royston P. Profile likelihood for estimation and confidence intervals. Stata Journal. 2007;7(3):376-387. doi: https://doi.org/10.1177/1536867X0700700305

27. Bull SB, Mak C, Greenwood CMT. A modified score function estimator for multinomial logistic regression in small samples. Comput Stat Data Anal. 2002;39(1):57-74. doi: https://doi.org/10.1016/S0167-9473(01)00048-2

28. Devika S, Jeyaseelan L, Sebastian G. Analysis of sparse data in logistic regression in medical research: a newer approach. J Postgrad Med. 2016;62(1):26-31. doi: https://doi.org/10.4103/0022-3859.173193

29. Henkle E, Aksamit T, Barker A, et al. Patient-centered research priorities for pulmonary nontuberculous mycobacteria (NTM) infection. An NTM research consortium workshop report. Ann Am Thorac Soc. 2016;13(9):S379-384.

doi: https://doi.org/10.1513/AnnalsATS.201605-387WS

30. Quittner AL, Madan A, Saez-Flores E, et al. Development of a quality of life module for nontuberculous mycobacteria (NTM). Eur Respir J. 2015;46(suppl 59):PA2635.

doi: https://doi.org/10.1183/13993003.congress-2015.PA2635

31. BoyleDP,ZembowerTR, Reddy S, QiC. Comparison of clinical features, virulence, and relapse among mycobacterium avium complex species. Am J Respir Crit Care Med. 2015;191(11):1310-1317. doi: https://doi.org/10.1164/rccm.201501-00670C

32. Lee BY, Kim S, Hong Y, et al. Risk factors for recurrence after successful treatment of mycobacterium avium complex lung disease. Antimicrob Agents Chemother (Bethesda). 2015;59(6):2972-2977. doi: https://doi.org/10.1128/AAC.04577-14

33. Min J, ParkJ, Lee YJ, et al. Determinants of recurrence after successful treatment of mycobacterium avium complex lung disease. Int JTuberc Lung Dis. 2015;19(10):1239-1245.

doi: https://doi.org/10.5588/ijtld.14.0139

34. Center for Drug Evaluation and Research, U.S. Food and Drug Administration (FDA). The voice of the patient: a series of reports from the U.S. Food and Drug Administration's (FDA's) PatientFocused Drug Development Initiative: non-tuberculous mycobacterial (NTM) lung infection. FDA website. Published April 2016. Accessed November 25, 2020. http://www.fda.gov/downloads/ForIndustry/ UserFees/PrescriptionDrugUserFee/UCM496941.pdf.

35. Quittner AL, Buu A, Messer MA, Modi AC, Watrous M. Development and validation of the cystic fibrosis questionnaire in the United States: a health-related quality-of-life measure for cystic fibrosis. Chest. 2005; 128(4):2347-2354.

doi: https://doi.org/10.1378/chest.128.4.2347

36. Quittner AL, Marciel KK, Salathe MA, et al. A preliminary quality of life questionnaire-bronchiectasis: a patient-reported outcome measure for bronchiectasis. Chest. 2014;146(2):437-448.

doi: https://doi.org/10.1378/chest.13-1891
37. Quittner AL, O'Donnell AE, Salathe MA, et al. Quality of life questionnaire-bronchiectasis: final psychometric analyses and determination of minimal important difference scores. Thorax. 2015;70(1):12-20.

doi: https://doi.org/10.1136/thoraxjnl-2014-205918

38. Center for Drug Evaluation and Research, U.S. Food and Drug Administration (FDA). Public meeting on non-tuberculous mycobacterial (NTM) lung infections patient-focused drug development. FDA website. Published October 15, 2015. Accessed November 25, 2020. https://www.fda.gov/media/130032/download.

39. Huang JH, Kao PN, Adi V, Ruoss SJ. Mycobacterium aviumintracellulare pulmonary infection in HIV-negative patients without preexisting lung disease: diagnostic and management limitations. Chest. 1999;115(4):1033-1040. doi: https://doi.org/10.1378/chest.115.4.1033

40. Field SK, Fisher D, Cowie RL. Mycobacterium avium complex pulmonary disease in patients without HIV infection. Chest. 2004;126(2):566-581. doi: https://doi.org/10.1378/chest.126.2.566

41. Kobashi Y, Matsushima T. The microbiological and clinical effects of combined therapy according to guidelines on the treatment of pulmonary mycobacterium avium complex disease in Japan including a follow-up study. Respiration. 2007;74(4):394-400. doi: https://doi.org/10.1159/000095674

42. O'Brien RJ, Geiter LJ, Dixie E, Snider J. The epidemiology of nontuberculous mycobacterial diseases in the United States. Am Rev Respir Dis. 1987;135(5):1007-1014. doi: https://doi.org/10.1164/arrd.1987.135.5.1007

43. Aliberti S, Sotgiu G, Castellotti P, et al. Real-life evaluation of clinical outcomes in patients undergoing treatment for non-tuberculous mycobacteria lung disease: a ten-year cohort study. Respir Med. 2020;164:105899.

doi: https://doi.org/10.1016/j.rmed.2020.105899

44. Park HY, Jeong BH, Chon HR, Jeon K, Daley CL, Koh WJ. Lung function decline according to clinical course in nontuberculous mycobacterial lung disease. Chest. 2016;150(6):1222-1232. doi: https://doi.org/10.1016/j.chest.2016.06.005

45. Khan K, Wang J, Marras TK. Nontuberculous mycobacterial sensitization in the United States. Am J Respir Crit Care Med. 2007;176(3):306-313. doi: https://doi.org/10.1164/rccm.200702-2010C 